\title{
Application of Multidisciplinary Design Optimization on Advanced Configuration Aircraft
}

\author{
Yalin Pan¹, Jun Huang1, Feng Li², Chuxiong Yan
}

\begin{abstract}
An optimization strategy is constructed to solve the aerodynamic and structural optimization problems in the conceptual design of double-swept flying wing aircraft. Aircraft preliminary aerodynamic and structural design optimization is typically based on the application of a deterministic approach of optimizing aerodynamic performance and structural weight. In aerodynamic optimization, the objective is to minimize induced drag coefficient, and the structural optimization aims to find the minimization of the structural weight. In order to deal with the multiple objective optimization problems, an optimization strategy based on collaborative optimization is adopted. Based on the optimization strategy, the optimization process is divided into system level optimization and subsystem level optimization. The system level optimization aims to obtain the optimized design which meets the constraints of all disciplines. In subsystem optimization, the optimization process for different disciplines can be executed simultaneously to search for the consistent schemes. A double-swept configuration of flying wing aircraft is optimized through the suggested optimization strategy, and the optimization results demonstrate the effectiveness of the method.
\end{abstract}

KEYWORDS: Aerodynamic performance, Structural optimization, Multiple objective optimization,

Collaborative optimization, Flying wing.

\section{INTRODUCTION}

Flying wing configuration has been considered as an ideal configuration of the future unmanned aerial vehicles (UAV) due to its potential benefits over conventional configurations in stealth capability, aerodynamic performance, and structural efficiency. Several next-generation UAVs are of flying wing designs, such as the X-45; X-47B; nEURO; etc. (Song et al. 2014; Li et al. 2014; Bolsunovsky et al. 2001).

Compared with the conventional configuration, flying wing aircraft has become the research hotspot of advanced aircraft in recent years (Zhou and Liu 2015), and the number of flying wing aircrafts which have been developed successfully is far less than the number of aircrafts with the conventional configuration. The lack of statistics and practical experience about flying wing configuration posed great difficulties in aircraft conceptual design. It has been proved that multidisciplinary design optimization (MDO) is an effective technique to deal with these problems. Now, it has been widely used in the conceptual design of traditional layout aircraft (Piperni et al. 2007; Lee et al. 2007; Lambe and Martins 2016).

Aircraft optimization problems involve multiple objectives and should be treated as multi-objective optimization (MOO) problems. As a classic example, aircraft preliminary aerodynamic and structural design optimization is typically based on the application of a deterministic approach of maximizing lift-to-drag ratio under cruising or other flight conditions and minimizing structural weight due to applied air load in an optimization process (Gou and Song 2006; Gao et al. 2003; Molinari et al. 2014). Thus, aerodynamic and structural 
optimization problem is a 2-objective optimization problem in aircraft design. In aerodynamic design, in order to minimize the induced drag coefficient, the shape of the lift coefficient distribution along the spanwise direction is expected to be close to an ellipse. However, under this kind of load distribution, the structural weight is not always the lightest during the process of structural design. Thus, in terms of load distribution, the aerodynamic performance and the structural weight are usually conflicting. In MOO, the optimization objectives belong to different disciplines always in conflict with each other. It is impossible to reach the optimal results for all the optimization goals at the same time. The optimization results ought to be a set of optimal solutions rather than an optimal solution. This set is known as the Pareto optimal set, and its corresponding tradeoff in objective space is known as the Pareto optimal frontier, which is made up of the Pareto optimal points (Sanghvi et al. 2014; Huang et al. 2007; Hu and Yu 2009). Designers could select an optimized scheme which satisfies the requirements of all disciplines from the Pareto optimal set. In this article, an optimization strategy based on collaborative optimization is proposed to deal with the MOO problem in flying wing aircraft conceptual design. The UAV configuration used as a basis for the MOO is shown in Fig. 1. The take-off weight of the aircraft is $20,000 \mathrm{~kg}$ and it is used for intelligence, surveillance, and reconnaissance missions as a high-altitude long-range UAV.

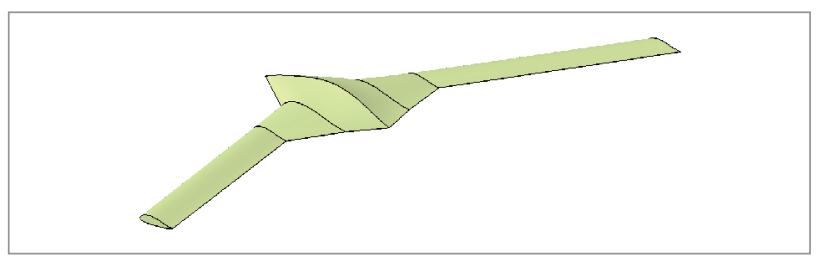

Figure 1. CAD model of flying wing aircraft.

\section{STATEMENT OF THE OPTIMIZATION PROBLEM}

The platform of the UAV is shown in Fig. 2. The parameters as shown in the figure are the outline ones and they are used to determine the planform of the UAV. The chord lengths at different locations along spanwise direction are described by $b_{r}$, $b_{2}$, and $b_{t}$. The wing span for different sections are described by $l_{1}, l_{2}$, and $l_{3}$. In order to describe the sectional shape of the UAV, section 1, section 2, section 3, and section 4 (as shown in Fig. 2) are defined as the master sections, whose profiles are displayed in Fig. 3 and Fig. 4; these sections are used to fit the contour surface of the aircraft. The lift and the induced drag of the UAV are mainly concerned with the airfoil camber. The outline parameters are fixed in the original design. It is expected that the minimum of the induced drag coefficient of the UAV can be found by adjusting the mean lines of these sections.

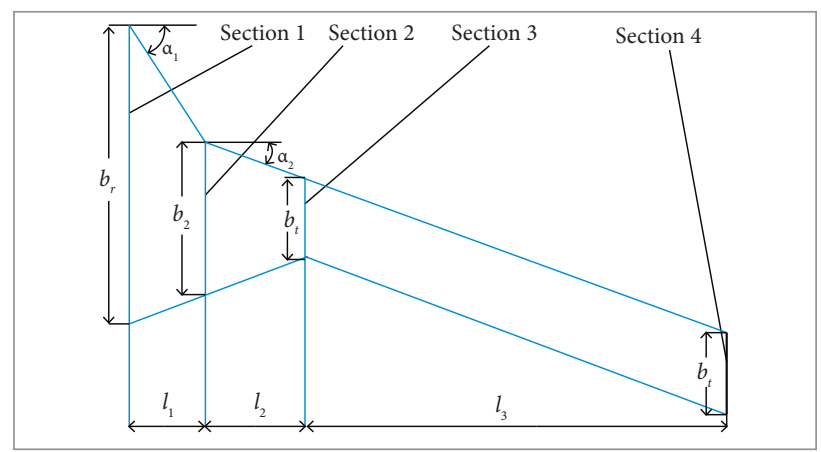

Figure 2. Half of flying wing configuration platform.

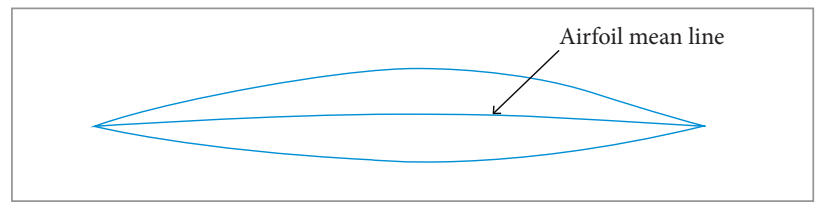

Figure 3. Profile of section 1.

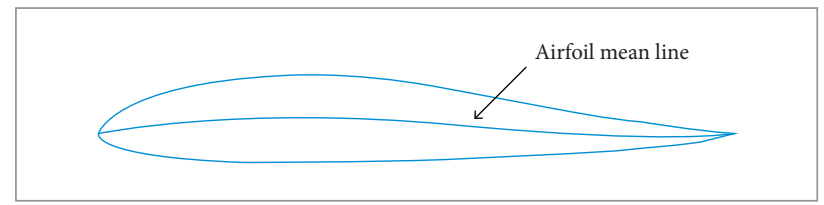

Figure 4. Profile of the other master sections.

Constrained by the material allowable stress and structural deformation, it is required to find the minimum of structural weight by adjusting the structural dimensions. The structure of the UAV contains outer skins and inner structural layout, as shown in Fig. 5. The inner structural layout of the UAV is displayed clearly in Fig. 6. For inner structure, the fuselage (Part 1) is composed by longitudinal beams and reinforced frames, the wing closed to the fuselage (Part 2) includes wing spars and reinforced ribs, and the outboard wing (Part 3 ) includes wing spars and wing ribs. The structural dimensions which are used as design variables include the thickness of skin and rib, the area of spar cap and rib cap, etc.

As already mentioned, the optimization problem of this UAV conceptual design can be formulated as follows:

- Objectives: (1) Minimized induced drag coefficient; (2) Minimized structural weight. 
- Design variables: (1) Parameters which are used to describe the airfoil mean lines of the UAV; (2) Parameters which are used to define the structure of the UAV.

- Constraints: (1) Aerodynamic requirements; (2) Structural requirements.

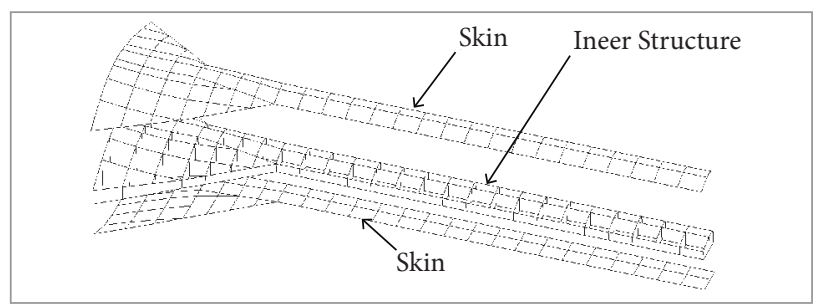

Figure 5. Structural layout of the UAV.

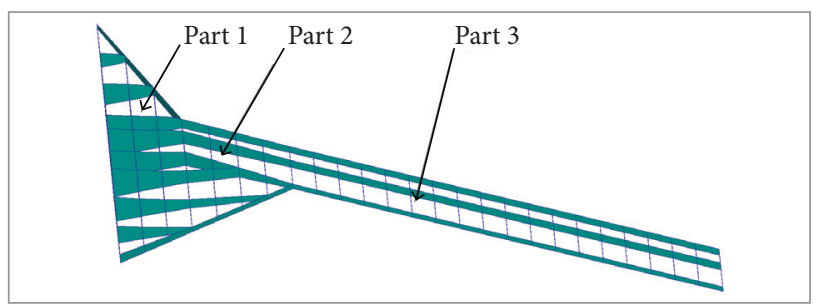

Figure 6. Inner structure of the UAV.

\section{OPTIMIZATION STRATEGY}

Aerodynamic and structural optimization problem is a typical MOO problem in aircraft design, and the 2 disciplines are always in conflict. The improvement of aerodynamic performance often brings an increase in structural weight, and the decrease in it usually causes the expense of aerodynamic performance (Chu 2011; Ma et al. 2009). In MOO, there often exists a set of optimal solutions, and none can be said to be better than any other without any further information. In order to coordinate the relationship between aerodynamic and structure of the UAV, an optimization strategy based on collaborative optimization is used to deal with the MOO problem. The optimization process can be divided into 2 levels which include the system level optimization and the subsystem level optimization. The system level optimization transfers the global design variables to the subsystem level optimization. In the subsystem level optimization, these global design variables are defined as target values. The state variables of each discipline are optimized to approximate these target values through optimization algorithm. Thus, the optimized designs for different disciplines are consistent.

The system level optimization aims to search for the design scheme with minimum induced drag coefficient and structural weight of the UAV. The constraint function of system optimization is used to make the schemes of different subsystem optimizations consistent. In this article, only the state variables of aerodynamic discipline which are used to describe the load distribution on the aircraft surfaces are defined as the global design variables (i.e. the design variables in system level optimization). The design parameters which only have impact to 1 discipline are defined as local design variables (i.e. the design variables in subsystem level optimization). Since the airfoil mean lines of the master sections have influence mainly on aerodynamic performance but have little effect on structure weight, the parameters which are used to describe the airfoil mean lines are local design variables. The structural dimensions have influence mainly on structure weight and impact on aerodynamic performance slightly, so these parameters are also local design variables. The load distribution can be described by lift coefficient curve and it is generated by fitting the lift coefficient of the sections along its spanwise direction. The lift coefficient curve is described by a cubic polynomial function. The fitting function is shown as follows:

$$
C l(\eta)=a_{3} \cdot \eta^{3}+a_{2} \cdot \eta^{2}+a_{1} \cdot \eta^{1}+a_{0}
$$

where: $\eta=y / b ; y$ represents the coordinates of the UAV spanwise direction; $b$ is half of the span; the shape of the load distribution curve is determined by the function coefficients (i.e. $a_{0}, a_{1}, a_{2}$, and $a_{3}$ ). The global design variables can be represented by $a_{0}, a_{1}, a_{2}$, and $a_{3}$.

The subsystem level optimization is integrated with the system level optimization, and the goal of aerodynamic optimization is to achieve the minimum of difference between the state variables and global design variables under the constraints of aerodynamic characteristics by changing the camber curve shapes of master sections airfoils. Constrained by the material allowable stress and structural deformation, the structure optimization aims to find the minimum of structural weight by adjusting the structural dimensions. The frame of the method for aerodynamic and structural multiple-objective design optimization is depicted in Fig. 7.

In the optimization strategy, the non-dominated sorting genetic algorithm (NSGA-ii) is adopted for system optimization. The formulation of system level optimization is stated as follows:

- Objectives: minimized induced drag coefficient $\left(C_{D i}\right)$ and structural weight $(W)$.

- Design variables: parameters which are used to define the lift coefficient distribution along spanwise direction, such as $a_{0} ; a_{1} ; a_{2}$ and $a_{3}$. 
- Constraints: the scheme optimized by the subsystem optimization is consistent with the design offered by the system $\left(J_{1}=0\right)$.

The sequential quadratic programming algorithm is used for aerodynamic optimization. The formulation of aerodynamic optimization problem is as follows:

- Given conditions: cruise Mach number is 0.8 , and cruise altitude is $18 \mathrm{~km}$.

- Objective: to minimize the difference between the state variables and the global design variables, $J_{1}=\sum_{i=0}^{3}\left(a_{i}-a_{i}^{0}\right)^{2}$

- Design variables: the parameters for describing the airfoil mean lines of master sections.

- $\quad$ Constraints: to design lift coefficient $\left(C_{L}=0.362\right)$.

The sequential quadratic programming algorithm is adopted for structural optimization. The formulation of the structural optimization problem is as follows:

- Objective: minimized structural weight $(W)$.

- Design variables: (1) the areas of spar caps, ribs, and reinforced frames; (2) the thicknesses of the webs of spars, ribs, and reinforced frames; (3) the thicknesses

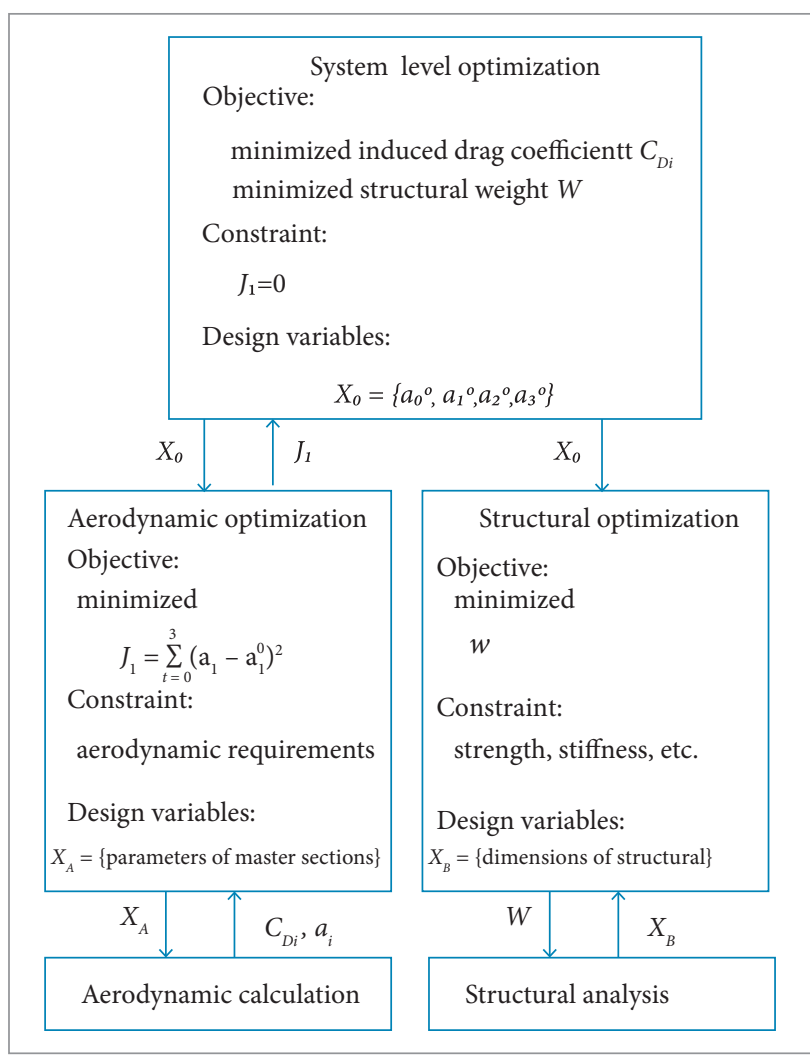

Figure 7. Optimization strategy for multi-objective optimization in aircraft conceptual design. of wing skins; (4) the stiffener areas of the webs. Eightysix dimensions in total are used as design variables.

- Constraints: (1) the axial stress of the rods $\leq 450 \mathrm{MPa}$; (2) the shear stress of the plates $\leq 250 \mathrm{MPa}$; (3) the displacement of wing tip $\leq 5 \%$ of the semi span of the wing.

The final result can be obtained by iterating the system level and the subsystem level optimizations until converging to the optimum values. The subsystem optimizations are executed as the global variables are updated.

\section{AIRCRAFT MODEL AND ANALYSIS METHODS}

Executing optimization process automatically is necessary for solving complex optimization problems. To implement the procedure of aerodynamic and structural optimization for the UAV conceptual design, some important technologies, like parametric geometry description and automatic execution of aerodynamic computing and structure calculation, are essential. These approaches which will be used in the optimization are explained in the following subsections.

\section{GENERATING PARAMETRIC MODEL}

As for all optimization tasks, the complexity of the problem is directly coupled to the parameterization of the geometry. Of highest relevance is the number of parameters that are required to ensure valid modeling. The most important characteristic of the CAD model is to be highly flexible in order to represent a variety of designs as large as possible. Secondly the model must be robust and reliable, since there will not be a specialist manually entering new parameters and supervising the update process (Amadori et al. 2008; Sripawadkul et al. 2010; Wang et al. 2013). Flying wing configuration has the characteristics of simple shape and blended wing body, and the UAV can be seen as a special wing which is connected together by 3 segments. The important content of the CAD model parameterization is to describe parametric airfoils shape of the UAV.

In the article, the airfoil profile is described by the superposition of camber distribution and thickness distribution. The function which is used to explain the camber distribution is shown as follows:

$$
\begin{aligned}
f(x) & =c_{1} \cdot \sin (\pi \cdot x)+c_{2} \cdot \sin \left(\pi \cdot x^{1.5}\right)+ \\
& +c_{3} \cdot \sin \left(\pi \cdot x^{2}\right)+c_{4} \cdot \sin \left(\pi \cdot x^{2.5}\right)
\end{aligned}
$$


The coefficients of the function are derived from four characteristic parameters of the airfoil mean line, such as relative camber $(C)$, relative camber location $(X C)$, the angle between leading edge of camber line and chord line $\left(\alpha_{L E}\right)$, and the angle between trailing edge of camber line and chord line $\left(\alpha_{T E}\right)$. The geometric significance of these parameters is shown in Fig. 8.

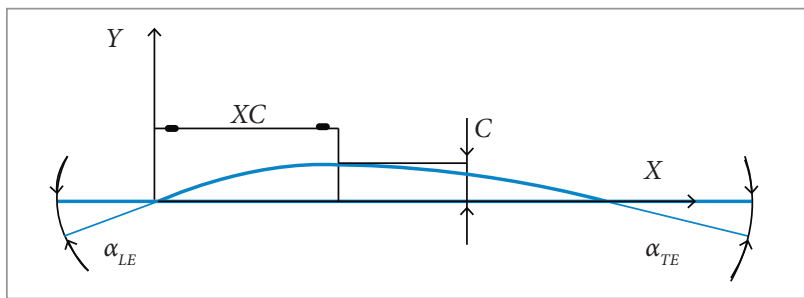

Figure 8. The meaning of camber parameters.

\section{AERODYNAMIC COMPUTING}

In order to get the induced drag coefficient and lift coefficient distribution along spanwise direction corresponding to design lift coefficient of the UAV, a panel code (Panair) is adopted. Panel codes are numerical schemes for solving the Prandtl-Glauert equation for linear, inviscid, irrotational flow about aircraft flying at subsonic or supersonic speeds (Lehmkuehler et al. 2012). Compared to CFD codes, Panair has advantages in terms of speed and ease of meshing. The surface mesh information of the UAV required for aerodynamic analysis is shown in Fig. 9. Pointwise software is used to divide quad surface mesh of the UAV model. An auxiliary numerical code is written to transform the surface mesh into the file which will be transmitted to Panair.

Aerodynamic performance is calculated through the following steps: (1) generating the mesh file of the UAV in Pointwise based on CAD model; (2) generating the file which will be transferred to Panair; (3) executing Panair program to calculate the lift coefficient of the UAV at different attack angles; (4) calculating the attack angle corresponding to design lift coefficient; (5) computing the aerodynamic performance of the UAV at design point; (6) deleting the files which are generated in the procedure.

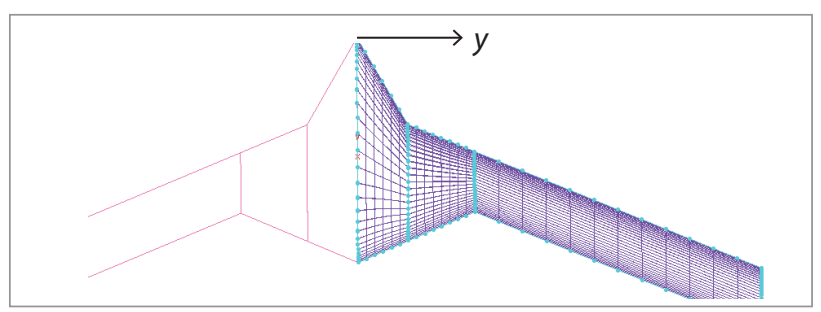

Figure 9. Aerodynamic model of the UAV.
Through aerodynamic performance calculation, the lift drag ratio and the lift coefficients of the sections along the UAV spanwise direction corresponding to design lift coefficient can be generated and outputted.

\section{STRUCTURAL ANALYSIS}

The structural model is more complex than the aerodynamic one due to the structural layout in the inertial model of the UAV. The features of the structural layout are defined by the number and positions of the spars, as well as the number and orientation of the ribs. These parameters are used for splitting the geometric model surface of the UAV in CATIA. Then the split model is transmitted to MSC.PATRAN to generate the structural layout model and the finite element model (FEM), which is shown in Fig. 10. In the FEM of the structure, the skins and webs of spars, ribs, and reinforced frames are modeled by plates, and the spars and the stiffeners for webs are modeled by rods. The dimension parameters of the FEM include thickness of skin and rib, area of spar caps and rib caps, etc.

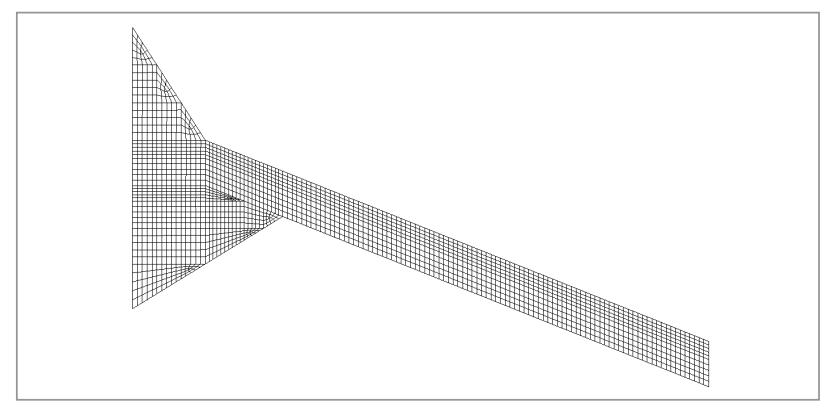

Figure 10. Finite element model for the UAV structure.

By using the FEM of the UAV structure, the optimization process can be used. The structural optimization is carried out by running MSC.NASTRAN software. It aims to search for the values of structural design variables which minimize structural weight in the condition when the material allowable stress, structural deformation, and geometry dimensions are satisfied. The structural weight optimized is necessary for overall performance calculation.

\section{OPTIMIZATION PROCESS}

In this article, iSIGHT is adopted to integrate all the software and programs we used (Koch et al. 2002). In order to improve the optimization efficiency, a surrogate model is constructed before MDO. Since aerodynamic problems are often highly non-linear, the surrogate model chosen in this article is 
the Radial Basis Function Neural Network (RBFNN). The input data of the surrogate model including the camber parameters of master sections and the output parameters are $a_{0} ; a_{1 ;} a_{2} ; a_{3}$ and $C_{D i}$. In the surrogate model, 370 sample points are selected randomly in design space to construct the surrogate model. Other 185 sample points are selected as the error analysis points of the surrogate model. In order to improve the credibility of the surrogate model, 370 new sample points are selected to reconstruct the surrogate model. The error analysis results of the final surrogate model for the output parameters are shown in Table 1. Six new sample points which are selected from the design space are calculated by Panair and the surrogated model, respectively, and the results are shown in Table 2. Once the surrogate model is built with given exact function data, it can efficiently be in the exploration of the design space and the MDO by replacing the original code.

Figure 11 shows the detailed procedure for dealing with the MOO problem of the UAV. The optimization process is carried out step by step following the flowchart. Each system level optimization iterates, during which the global variables can be updated and then the subsystem level optimization is completed once.

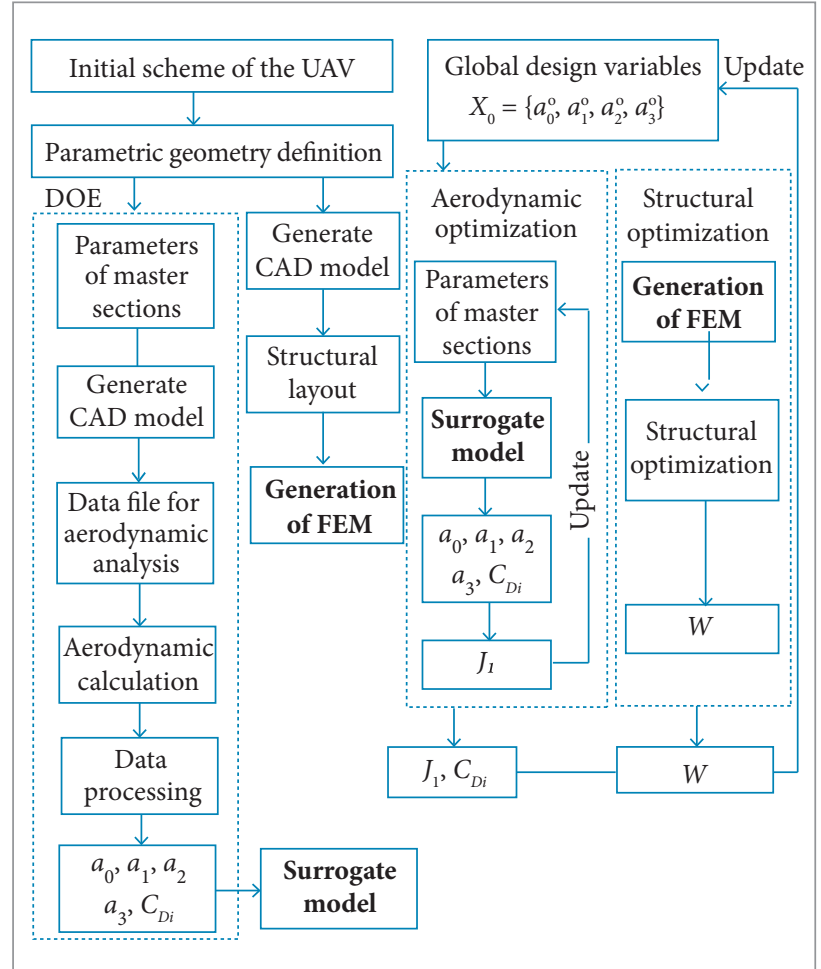

Figure 11. Flowchart of aerodynamic and structural multidisciplinary design optimization.

Table 1. Approximation error analysis.

\begin{tabular}{|c|c|c|c|c|}
\hline Variables & Average & Maximum & Root Mean Square & R-squared \\
\hline$a_{0}$ & 0.00508 & 0.02527 & 0.00677 & 0.99909 \\
\hline$a_{1}$ & 0.00456 & 0.02435 & 0.006 & 0.99912 \\
\hline$a_{2}$ & 0.00423 & 0.02318 & 0.00551 & 0.99911 \\
\hline$a_{3}$ & 0.00423 & 0.02274 & 0.00545 & 0.99909 \\
\hline$C_{D i}$ & 0.0134 & 0.04002 & 0.01572 & 0.9927 \\
\hline
\end{tabular}

Table 2. Comparison of calculation results by Panair and RBFNN model.

\begin{tabular}{|c|c|c|c|c|c|c|c|}
\hline \multicolumn{2}{|c|}{ Responses } & 1 & 2 & 3 & 4 & 5 & 6 \\
\hline \multirow{2}{*}{$a_{0}$} & Panair & 2.028481 & 2.135833 & 2.099838 & 2.129787 & 2.089403 & 2.056113 \\
\hline & RBFNN & 2.029884 & 2.135446 & 2.097253 & 2.130500 & 2.092359 & 2.056392 \\
\hline \multirow[b]{2}{*}{$a_{1}$} & Panair & -3.284242 & -3.56747 & -3.718978 & -3.458064 & -3.533423 & -3.257147 \\
\hline & RBFNN & -3.287965 & -3.56777 & -3.70466 & -3.461285 & -3.549795 & -3.262633 \\
\hline \multirow{2}{*}{$a_{2}$} & Panair & 2.760191 & 2.858455 & 3.431695 & 2.571950 & 3.064149 & 2.578853 \\
\hline & RBFNN & 2.760020 & 2.860046 & 3.409363 & 2.578452 & 3.089931 & 2.589869 \\
\hline \multirow{2}{*}{$a_{3}$} & Panair & -1.422091 & -1.35417 & -1.733668 & -1.181949 & -1.54088 & -1.298712 \\
\hline & RBFNN & -1.419876 & -1.35518 & -1.72302 & -1.185902 & -1.553252 & -1.304347 \\
\hline \multirow{2}{*}{$C_{D i}$} & Panair & 0.003510 & 0.003480 & 0.003470 & 0.003410 & 0.003470 & 0.003480 \\
\hline & RBFNN & 0.003505 & 0.003478 & 0.003472 & 0.003405 & 0.003473 & 0.003477 \\
\hline
\end{tabular}




\section{OPTIMIZATION RESULTS}

Based on the given optimization strategy, the optimization process is executed automatically. Figure 12 provides the Pareto fronts which are the solutions of minimizing both the induced drag coefficient and structural weight. An optimal point can be selected from the Pareto optimal solution set according to the design requirements.

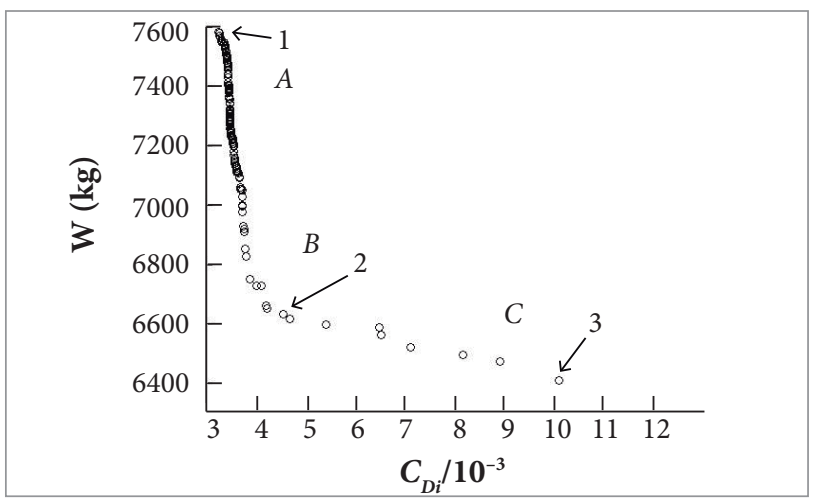

Figure 12. Pareto optimal front obtained from system level optimization.

The Pareto optimal front can be divided into 3 typical subsets, including subset $A$, subset $B$, and subset $C$. These subsets correspond to 3 typical subsets of the Pareto optimal solution set, respectively. The subset $\mathrm{A}$ is a set of optimization solutions with smaller induced drag coefficient. The subset $C$ represents the optimization solutions which have lighter structural weight. The subset $B$ lying between subsets $\mathrm{A}$ and $\mathrm{C}$ is the trade-off between aerodynamic performance and structural weight, so it has both relatively smaller induced drag coefficient and lighter structural weight. The choice for a solution from each subset as the typical sample and the aerodynamic performance and structural weight of selected samples are listed in Table 3, which shows that the aerodynamic
Table 3. Typical solutions in Pareto optimal front.

\begin{tabular}{|c|c|c|}
$\begin{array}{c}\text { Number of the } \\
\text { sample }\end{array}$ & $C_{D i}$ & $W[\mathrm{~kg}]$ \\
\hline 1 & 0.00323511 & 7,582 \\
\hline 2 & 0.00454684 & 6,632 \\
\hline 3 & 0.01012 & 6,409 \\
\hline
\end{tabular}

performance of design 1 is better than the 2 other solutions; design 3 is the best one with respect to structural weight; and design 2 is a solution which is a compromise of the induced drag coefficient and structural weight.

\section{CONCLUSION}

In this paper, an investigation has been made to study aerodynamic and structural optimization of flying wing aircraft. A multi-objective optimization strategy based on collaborative optimization strategy is proposed. During the optimization process, the parallel computing in subsystem optimization is used, which improves the efficiency of optimization. The optimization result is a Pareto optimal set, which provides the designer with more options. A flying wing configuration of aircraft is optimized by the strategy, and the optimization results demonstrate that the present method can efficiently find the Pareto optimal set.

\section{AUTHORS' CONTRIBUTIONS}

Pan Y and Huang J conceived the idea and co-wrote the main text; Li F and Yan C constructed the numerical models for multidisciplinary analysis; Yan $\mathrm{C}$ processed the calculation data. All authors discussed the results and commented on the manuscript. This paper was carried out through the collaboration of all authors.

\section{REFERENCES}

Amadori K, Jouannet C, Krus P (2008) Aircraft conceptual design optimization. Proceedings of the 28th Congress of the International Council of the Aeronautical Sciences; Anchorage, USA.

Bolsunovsky AL, Buzoverya NP, Gurevich BI, Denisov VE, Dunaevsky Al, Shkadov LM, Sonin OV, Udzhuhu AJ, Zhurihin JP (2001) Flying wing problems and decisions. Aircraft Des 4(4):193-219. doi: 10.1016/ S1369-8869(01)00005-2

Chu $L$ (2011) Integration design of aerodynamic, structure and stealthy performance for joined-wing. Aeronautical Science and Technology 2:57-60.

Gao ZH, Xia L, Li T, Zhao X(2003) Investigation into collaborative optimization design techniques of aircraft aerodynamics and stealth performances. Aircraft Des 3:1-5. doi: 10.3969/j.issn.1673-4599.2003.03.001

Gou ZQ, Song BF (2006) The research of structural optimization with the constraints of aerodynamics and radar cross section (RCS) of aircraft. Systems Engineering - Theory \& Practice 3:135-140. 
Hu TY, Yu XQ (2009) Aerodynamic/stealthy/structural multidisciplinary design optimization of unmanned combat air vehicle. Chin J Aeronaut 22(4):380-386. doi: 10.1016/S1000-9361(08)60114-4

Huang CH, Galuski J, Bloebaum CL (2007) Multi-objective Pareto concurrent subspace optimization for multidisciplinary design. AIAA J 45(8):1894-1906. doi: 10.2514/1.19972

Koch PN, Evans JP, Powell D (2002) Interdigitation for effective design space exploration using iSIGHT. Structural and Multidisciplinary Optimization 23(2):111-126. doi: 10.1007/s00158-002-0171-9

Lambe AB, Martins JR (2016). Matrix-free aerostructural optimization of aircraft wings. Structural and Multidisciplinary Optimization 53(3):589-603. doi: 10.1007/s00158-015-1349-2

Lee D, Gonzalez LF, Srinivas K, Auld D, Periaux J (2007) Multiobjective/multidisciplinary design optimisation of blended wing body UAV via advanced evolutionary algorithms. Proceedings of the 45th AIAA Aerospace Sciences Meeting; Reno, USA.

Lehmkuehler K, Wong K, Verstraete D (2012) Design and test of a UAV blended wing body configuration. Proceedings of the 28th Congress of the International Council of the Aeronautical Sciences; Brisbane, Australia.

Li YH, Huang Y, Su JC (2014) Aerodynamic analysis and design numerical simulation of the vortex flow over a common research model with flying-wing configuration. Appl Mech Mater 540:138-142. doi: 10.4028/www.scientific.net/AMM.540.138

Ma TL, Ma DL, Zhang S (2009) Method for aerodynamic and structure integrative design. Journal of Beijing University of Aeronautics and Astronautics 35(11): 1366-1383. doi: 10.13700/j.bh.10015965.2009.11.011

Molinari G, Arrieta AF, Ermanni P (2014) Aero-structural optimization of three-dimensional adaptive wings with embedded smart actuators. AIAA J 52(9):1940-1951. doi: 10.2514/1.J052715

Piperni P, Abdo M, Kafyeke F, Isikveren AT (2007) Preliminary aerostructural optimization of a large business jet. $J$ Aircraft 44(5):1422-1438. doi: 10.2514/1.26989

Sanghvi RC, Vashi AS, Patolia HP, Jivani RG (2014) Multi-objective optimization of two-stage helical gear train using NSGA-II. Journal of Optimization 2014:1-8. doi: 10.1155/2014/670297

Song L, Yang H, Zhang $Y$, Zhang H, Huang J (2014) Dihedral influence on lateral-directional dynamic stability on large aspect ratio tailless flying wing aircraft. Chin J Aeronaut 27(5):1149-1155. doi: 10.1016/j.cja.2014.08.003

Sripawadkul V, Padulo M, Guenov M (2010) A comparison of airfoil shape parameterization techniques for early design optimization. Proceedings of the 13th AIAA/ISSMO Multidisciplinary Analysis and Optimization Conference; Fort Worth, USA.

Wang WJ, Huang J, Song L (2013) A geometric representation of airfoil based on applied parameters. Aircraft Des 33(3):1-4.

Zhou HX, Liu B (2015) Characteristics analysis and optimization of flying-wing vehicle structure. Adv Mater Res 1077:177-184. doi: 10.4028/www.scientific.net/AMR.1077.177 